\title{
Lectin-Based Homogeneous Enzyme-Linked Binding Assay for Estimating the Type and Relative Amount of Carbohydrate within Intact Glycoproteins
}

\author{
Bokhee Kim, Iraj Behbahani, ${ }^{1}$ and Mark E. Meyerhoff ${ }^{2}$ \\ Department of Chemistry, The University of Michigan, Ann Arbor, Michigan 48109
}

Received September 6, 1991

\begin{abstract}
The feasibility of using a new lectin-based homogeneous enzyme-linked binding assay for estimating the type and relative amount of specific carbohydrate structures within intact glycoproteins is examined. Malate dehydrogenase-galactose, -mannose, and $-\boldsymbol{N}$-acetylglucosamine conjugates are utilized in conjunction with Jacalin, concanavalin $A$, and wheat germ agglutinin, respectively. The catalytic activity of the glyco-enzyme conjugates is inhibited significantly $(>60 \%)$ in solution in the presence of the respective lectins. The observed inhibition for each reagent set is reversed in proportion to the type and relative amount of specific carbohydrates present within test glycoproteins added to the assay mixture. Competitive binding $\mathbf{E D}_{50}$ values for a number of synthetic and native model glycoproteins correlate well with the known carbohydrate content of these species. The proposed method is much faster than previous solid-phase lectin-based enzymelinked methods used to probe carbohydrate content/ structure $(<15 \mathrm{~min})$ and has the potential to be fully automated. (1) 1992 Academic Press, Inc.
\end{abstract}

Recent application of advanced biotechnology methods to produce specific glycoproteins for therapeutic purposes (e.g., tissue plasminogen activator, etc.) has created a demand for additional analytical methods to characterize the carbohydrate content of such recombinant DNA products (1). While the amino acid sequence of these proteins can be fixed, glycosylation within given cell cultures can vary, and such changes in glycosylation can greatly affect the biological activity of the resulting product. Although a variety of complex instrumental

\footnotetext{
${ }^{1}$ Department of Chemistry, University of Michigan-Flint Campus Flint, MI.

${ }^{2}$ To whom correspondence should be addressed.
}

methods (e.g., mass spectrometry, NMR, etc.) have been utilized to determine the definitive carbohydrate structure of proteins (2-4) (often after tedious chemical and enzymatic digestions), there is also an immediate need for relatively simple and rapid methods that can, at the very least, detect significant changes in the type and relative amount of specific carbohydrates appended to the proteins. The unique carbohydrate binding specificity of lectins may be useful in this regard, and methods based on serial affinity chromatography with different immobilized lectins have been suggested (5-7). In addition, various enzyme-labeled lectins have been employed recently as carbohydrate probes in solid-phase assay arrangements (8-10). The glycan detection kits commercialized by Boehringer-Mannheim (Indianapolis, IN) function similarly in that the final signal is observed after addition of enzyme substrate, although the lectins are actually linked to digoxigenin and enzymelabeled anti-digoxigenin is used to detect bound lectin. Unfortunately, all of these previously reported enzymelinked methods are heterogeneous systems requiring extensive washing steps to separate bound and free enzyme-labeled species. We recently described a novel homogeneous enzyme-linked binding assay (ELBA) ${ }^{3}$ system for studying the interactions of lectins with carbohydrates and glycoproteins (11). The purpose of the present report is to demonstrate the feasibility of using an array of these new homogeneous enzymelinked/lectin reagents for estimating the type and relative amount of specific carbohydrate species present within intact glycoproteins.

Homogeneous enzyme-linked binding assays (e.g., enzyme-multiplied immunoassay technique (EMIT)

\footnotetext{
${ }^{3}$ Abbreviations used: ELBA, enzyme-linked binding assay; EMIT enzyme-multiplied immunoassay technique; $\mathrm{MDH}$, malate dehydrogenase; Con A, concanavalin A; WGA, wheat germ agglutinin.
} 


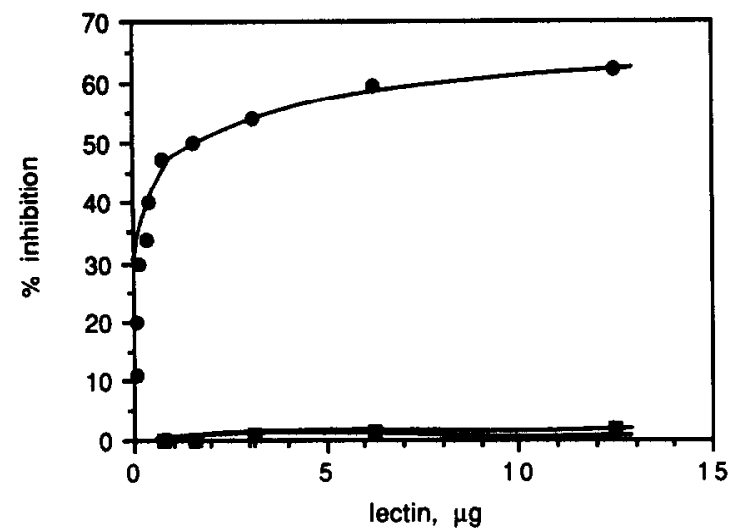

FIG. 1. Effect of varying amount of lectins on the catalytic activity of $\mathrm{MDH}-\mathrm{glcNAc}$ conjugate (conjugate concentration: $1.5 \times 10^{-9} \mathrm{M}$ in a $100-\mu$ l aliquot); (๑) WGA, (口) Con A, (+) Jacalin.

and the newer CEDIA (presumably competitive enzyme donor immunoassay) type enzyme immunoassays $(12,13))$ are attractive bioanalytical methods because there are no time-consuming washing or incubation steps associated with their use. In fact, all the components of the assay are mixed in a single homogeneous solution phase within a tube or cuvette prior to determining the catalytic activity of the enzyme label (by subsequent addition of substrate). This greatly enhances the speed of the assays and enables relatively simple automation of such methods. While homogeneous assays have been successfully devised to detect a variety of low-molecular-weight analytes (e.g., drugs, vitamins, etc.), there has been only limited success in adapting this attractive concept for the detection of large proteins (14-16).

In our previous work (11), we found that the catalytic activity of appropriately prepared monosaccharide conjugates of malate dehydrogenase (MDH) and glucose6-phosphate dehydrogenase could be inhibited (up to $70 \%$ ) in solution by lectins that bound strongly to the appended saccharide structures. When carbohydrates or glycoproteins recognized by the given lectin were also added to the assay mixture, enzyme activity increased in an amount proportional to the concentration of carbo- hydrate or glycoprotein present. The concentration required to reverse the inhibition was dependent on the relative binding affinity of the lectin toward the added species. For example, it was observed that galactoserich human IgA (immunoglobulin A) bound to Jacalin lectin tightly enough to fully reverse inhibition of $\mathrm{MDH}$-galactose conjugates at relatively low levels of the protein $(10 \mu \mathrm{g} / \mathrm{ml})$, while human IgG or IgM did not (i.e., no response to these proteins at $2 \mathrm{mg} / \mathrm{ml}$ levels). This result stimulated us to pursue the studies described herein.

Specifically, in this paper, three different lectin/enzyme-saccharide systems are utilized in a "fingerprint" type assay scheme to determine whether there is a direct correlation between the observed homogeneous doseresponse behavior (e.g., $\mathrm{ED}_{50}$ values) and the type and relative amount of certain carbohydrate structures within given glycoproteins. The array of systems used include: Conconavalin A (Con A) with a MDH-mannose conjugate; Jacalin with a MDH-galactose conjugate; and wheat germ agglutinin (WGA) with an $\mathrm{MDH}-\mathrm{N}$-acetylglucosamine conjugate. Synthetic BSAsaccharide conjugates with varying degrees of saccharide substitution and several native glycoproteins with known carbohydrate content are used as model analytes. It will be shown that the responses of each homogeneous lectin/enzyme-conjugate system correlate reasonably well with the known type and relative amount of carbohydrate present within the glycoproteins examined.

\section{MATERIALS AND METHODS}

Apparatus. Enzymatic activities were measured with a Gilford-Stasaar-III spectrophotometer equipped with a vacuum-operated sampling system and temperature-controlled cuvette (maintained at $30^{\circ} \mathrm{C}$ throughout the experiments). This spectrophotometer was connected to a Syva CP-5000 EMIT clinical processor for automatically setting the reading intervals and recording the absorbance values. A Perkin-Elmer $\lambda$ Array $3840 \mathrm{uv} / V i s$ spectrophotometer operated by a Model 7300 professional computer was used to obtain the spec-

TABLE 1

Effect of Monosaccharide Substitution on $\mathrm{ED}_{50}$ Values of BSA-Saccharide Conjugates in Two Different Lectin-Based Homogeneous ELBA Systems

\begin{tabular}{ccccc} 
& \multicolumn{2}{c}{ Jacalin/MDH-galactose } & \multicolumn{2}{c}{ Con A/MDH-mannose } \\
\cline { 2 - 3 } $\begin{array}{c}\text { Initial ratios of } \\
\text { ligand to BSA }\end{array}$ & $\begin{array}{c}\text { Degree of galactose } \\
\text { substitution }\end{array}$ & $\begin{array}{c}\mathrm{ED}_{50} \\
(\mu \mathrm{g} / \mathrm{ml})\end{array}$ & & $\begin{array}{c}\text { Degree of mannose } \\
\text { substitution }\end{array}$ \\
\hline 100 & 9.6 & 16.7 & 7.9 & $\begin{array}{c}\mathbf{E D}_{50} \\
(\mu \mathrm{g} / \mathrm{ml})\end{array}$ \\
250 & 13.4 & 8.1 & 12.5 & 23.7 \\
500 & 28.5 & 2.8 & 18.9 & 15.9 \\
\hline
\end{tabular}


TABLE 2

$\mathrm{ED}_{50}$ Values for Mixed BSA-Saccharide Conjugates Containing Varying Ratios of Mannose and Galactose Obtained with Two Different Lectin-Based Homogeneous ELBA Systems

\begin{tabular}{|c|c|c|}
\hline \multirow{2}{*}{$\begin{array}{c}\text { Initial ratios of } \\
\text { mannose/galactose }\end{array}$} & \multicolumn{2}{|c|}{$\mathrm{ED}_{50}(\mu \mathrm{g} / \mathrm{ml})$} \\
\hline & Jacalin/MDH-gal & Con $\mathrm{A} / \mathrm{MDH}-\operatorname{man}$ \\
\hline $1 / 3$ & 1.9 & 33.7 \\
\hline $1 / 1$ & 4.8 & 19.0 \\
\hline $3 / 1$ & 10.7 & 6.7 \\
\hline
\end{tabular}

tra of enzyme and enzyme-ligand conjugates to determine the degree of saccharide conjugation. A microdialyzer System 500 from Pierce was used for dialyzing the enzyme-saccharide conjugates.

Reagents. $\quad \beta$-Fructofuranosidase, human $\alpha_{1}$-acid glycoprotein, $\alpha$-amylase (from aspergillus oryzae), glucose oxidase, WGA, Con A, bovine submaxillary mucine, fetuin, $p$-isothiocyanatophenyl - $\alpha-\mathrm{D}$ - mannopyranoside, $p$ - isothiocyanatophenyl - $\alpha$ - D - galactopyranoside, $p$ isothiocyanato $-\beta-\mathrm{D}-N$-acetylglucosamine, and $\mathrm{MDH}$ (from pigeon breast muscle) were all obtained from Sigma Chemical Co. (St. Louis, MO). The MDH substrates, oxaloacetic acid and $\mathrm{NADH}$, were also Sigma products. Jacalin was purchased from Pierce (Rockford, IL). The assay for MDH and MDH-saccharide conjugate activity was performed in $0.10 \mathrm{M}$ sodium phosphate buffer, pH 7.5, containing $0.01 \%(\mathrm{w} / \mathrm{v}) \mathrm{NaN}_{3}$ and $0.30 \%$ $(w / v)$ gelatin (assay buffer). Deionized water was used to prepare all buffers.

Preparation of BSA-saccharide and $M D H$-saccharide conjugates. The MDH-galactose (MDH-gal), MDHmannose (MDH-man), $\mathrm{MDH}-\mathrm{N}$-acetylglucosamine (MDH-glcNAc), and BSA-saccharide conjugates used in this work were prepared as described previously (11). Briefly, $p$-isothiocyanatophenyl-D-glycoside ( $\alpha$-mannopyranoside, $\alpha$-galactopyranoside, or $\beta-N$-acetylglucosamine) was added in small portions to BSA or $\mathrm{MDH}$ so-

TABLE 3

Characteristics of Glycoproteins Examined

\begin{tabular}{lcccc}
\hline & & \multicolumn{3}{c}{ wt\% carbohydrate } \\
\cline { 4 - 5 } \multicolumn{1}{c}{ Glycoprotein } & $\begin{array}{c}\text { Molecular } \\
\text { weight }\end{array}$ & Gal & Man & GlcNAc \\
\hline \multirow{2}{*}{$\beta$-Fructofuranosidase } & 270,000 & $-\bar{b}^{b}$ & $50-77$ & $3.0-4.0$ \\
$\alpha_{1}$-Acid glycoprotein & 40,800 & $6.0-11$ & $5.0-6.0$ & $12-15$ \\
$\alpha$-Amylase & 51,000 & 0.35 & $2.1-2.5$ & 0.87 \\
Glucose oxidase & 180,000 & 0.30 & 14 & 2.0 \\
\hline
\end{tabular}

a Ref. (20).

${ }^{b}$ Essentially 0 wt\%.

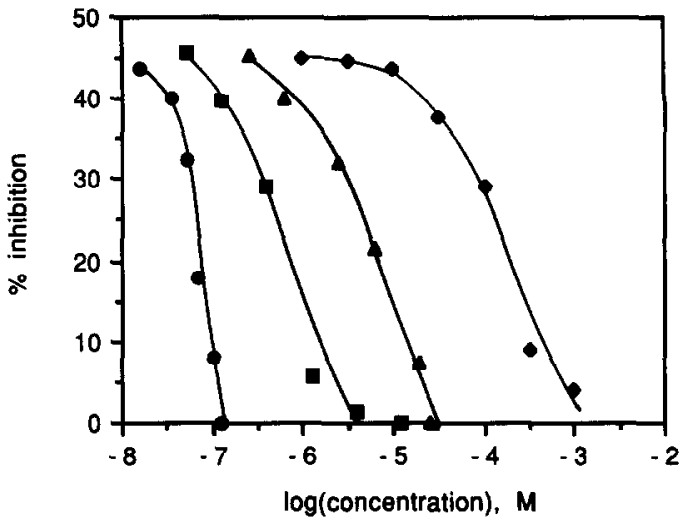

FIG. 2. Glycoprotein dose-response curves with MDH-mannose conjugate (Con A, $13.6 \mu \mathrm{g}$; conjugate concentration, $1.5 \times 10^{-9} \mathrm{M}$ in a

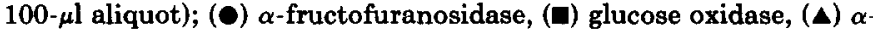
amylase, $(\diamond) \alpha_{1}$-acid glycoprotein.

lutions $(0.6 \mathrm{mg} / \mathrm{ml})$ buffered at $\mathrm{pH} 9.0\left(\mathrm{HCO}_{3}^{-} / \mathrm{CO}_{3}^{2-}\right)$. The coupling reaction was run for $24 \mathrm{~h}$ at $4^{\circ} \mathrm{C}$ with stirring. After extensive dialysis against phosphate buffer, the resulting conjugates were characterized by their residual activities, degree of saccharide conjugation, and percentage inhibition induced by excess amount of lectins. The degree of conjugation was determined by the phenol-sulfuric acid method for BSA-saccharide conjugates (17) and the Morgan-Elson method for MDH$N$-acetylglucosamine conjugates (18).

Determination of enzymatic activity and maximum percentage inhibition. The activity of $\mathrm{MDH}$-saccharide conjugates was determined by measuring the rate of decrease of NADH absorbance at $340 \mathrm{~nm}$ after addition of $100 \mu \mathrm{l}$ of NADH $\left(6.4 \times 10^{-4} \mathrm{M}\right.$ in $0.10 \mathrm{~mol} / \mathrm{liter}$ phosphate-gel assay buffer)), $100 \mu \mathrm{l}$ of oxaloacetic acid (1.9 $\times 10^{-3} \mathrm{M}$ in phosphate-gel assay buffer), and $100 \mu \mathrm{l}$ of a

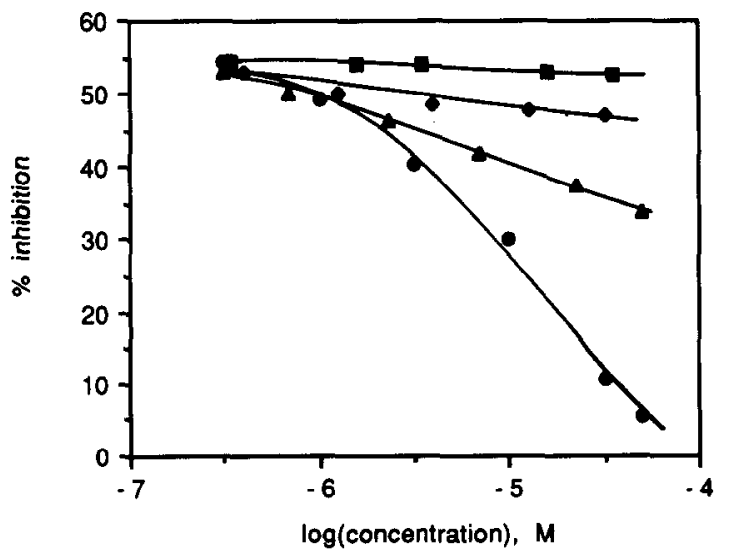

FIG. 3. Glycoprotein dose-response curves with $\mathrm{MDH}$-galactose conjugate (Jacalin, $5 \mu \mathrm{g}$; conjugate concentration, $1.5 \times 10^{-9} \mathrm{M}$ in a $100-\mu \mathrm{l}$ aliquot); (৩) $\alpha_{1}$-acid glycoprotein, (४) $\alpha$-amylase, ( $(\diamond)$ glucose oxidase, ( $\square$ ) $\beta$-fructofuranosidase. 
TABLE 4

$\mathrm{ED}_{50}$ Values of Glycoproteins Obtained with Three Different Lectin-Based Homogeneous ELBA Systems

\begin{tabular}{|c|c|c|c|}
\hline \multirow[b]{2}{*}{ Glycoprotein } & \multicolumn{3}{|c|}{$\mathrm{ED}_{50}(\mathrm{mg} / \mathrm{ml})$} \\
\hline & Jacalin/MDH-gal & Con $\mathrm{A} / \mathrm{MDH}-\operatorname{man}$ & WGA/MDH-glcNAc \\
\hline$\alpha_{1}$-Acid glycoprotein & 0.51 & 5.1 & 0.16 \\
\hline$\alpha$-Amylase & $>2.4^{a}$ & 1.0 & $>2.4^{a}$ \\
\hline Glucose oxidase & $>4.6^{a}$ & 0.073 & 1.5 \\
\hline
\end{tabular}

${ }^{a}$ Value indicated was highest concentration tested and this level did not reverse inhibition significantly, i.e., still less than true $\mathrm{ED}_{50}$ value.

properly diluted $\mathrm{MDH}$-saccharide conjugate (usually 1:1000 dilution) to a gelatin-coated disposable plastic sample tube containing $700 \mu \mathrm{l}$ of assay buffer. All solutions were kept at $0^{\circ} \mathrm{C}$ until the addition of the reagents. For each assay, after mixing reagents and subsequent agitation on a vibrator for a few seconds, the reaction mixture was aspirated into the thermostated flow cell of the spectrophotometer and the absorbance at $340 \mathrm{~nm}$ was measured over 1-min period after an initial delay of $20 \mathrm{~s}\left(\Delta A \min ^{-1}\right)$.

In order to determine the maximum percentage inhibition value for each conjugate, $100 \mu \mathrm{l}$ of the assay buffer was replaced by $100 \mu \mathrm{l}$ of the appropriate lectin solution prepared in assay buffer. In addition, the conjugates were incubated first with the lectin for 10-15 min before addition of the substrate solutions.

Kinetics of association between $M D H-g l c N A c$ conjugate and WGA. A WGA solution $(100 \mu \mathrm{l}, 100 \mu \mathrm{g} / \mathrm{ml})$ in assay buffer was incubated for varying periods of time with $100 \mu \mathrm{l}$ of conjugate $\left(1.5 \times 10^{-9} \mathrm{M}\right)$ in the same buffer. After each incubation period, enzymatic activity was determined as described above.

Effect of varying amount of lectin on the catalytic activity of MDH-glcNAc conjugate. Portions of solutions

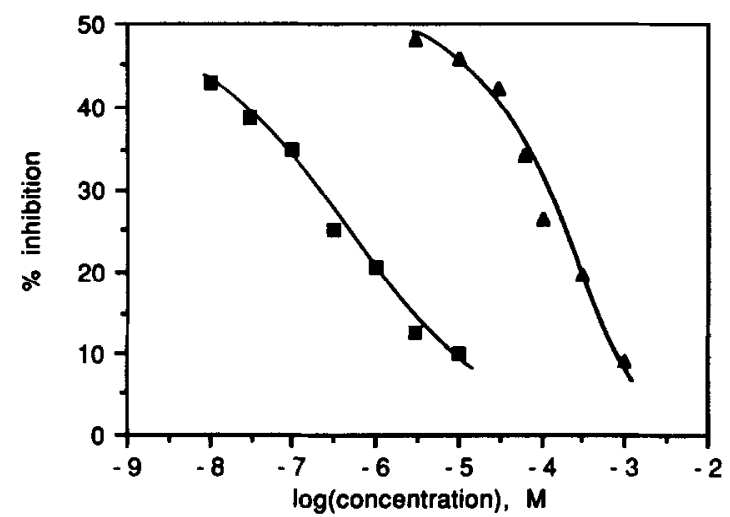

FIG. 4. Dose-response curves for sialic acid-rich glycoproteins with WGA/MDH-glcNAc conjugate (WGA, $1 \mu \mathrm{g}$; conjugate concentration, $1.5 \times 10^{-9} \mathrm{M}$ in a $100-\mu$ l aliquot); (0) bovine submaxillary mucin, (A) fetuin.
$(100 \mu \mathrm{l})$ containing different amounts of lectin were incubated with $100 \mu \mathrm{l}$ of conjugate $\left(1.5 \times 10^{-9} \mathrm{M}\right)$ for 10 min. Enzymatic activity was then measured as outlined above.

Dose-response curves for various glycoproteins with different carbohydrate content. Standard solutions of different glycoproteins were prepared in assay buffer. In the assay protocol, $100 \mu \mathrm{l}$ of standard solution and 100 $\mu$ of enzyme-saccharide conjugate solution were added to a gelatin-coated disposable tube containing $100 \mu \mathrm{l}$ of Con A $(133 \mu \mathrm{g} / \mathrm{ml}), 100 \mu \mathrm{l}$ of Jacalin $(50 \mu \mathrm{g} / \mathrm{ml})$, or $100 \mu \mathrm{l}$ of WGA lectin $(10 \mu \mathrm{g} / \mathrm{ml})$ solution. The mixtures were incubated for 10 or $15 \mathrm{~min}$ on a shaker. The resulting enzymatic activity was measured as described above. Dose-response curves were prepared by plotting average (for duplicate tubes) percentage inhibition vs the logarithm of the concentration of each glycoprotein analyte in the $100 \mu \mathrm{l}$ of standard solutions added to the assay mixture. The $\mathrm{ED}_{50}$ values (i.e., the concentration of glycoprotein required to achieve $50 \%$ of the total percentage inhibition value for a given set of reagents) were determined graphically from the dose-response curves.

\section{RESULTS AND DISCUSSION}

We have reported previously on the synthesis and lectin-induced inhibition of a variety of enzyme-man-

TABLE 5

Comparison of $\mathrm{ED}_{50}$ Values of Homogeneous ELBA Using MDH-glcNAc Conjugate/WGA System and Agglutinin Method

\begin{tabular}{lcc}
\hline & \multicolumn{2}{c}{$\operatorname{ED}_{50}(\mu \mathrm{M})$} \\
\cline { 2 - 3 } \multicolumn{1}{c}{$\begin{array}{c}\text { Saccharide or } \\
\text { glycoprotein }\end{array}$} & $\begin{array}{c}\text { Homogeneous } \\
\text { ELBA }\end{array}$ & Agglutinin $^{a}$ \\
\hline$N$-Acetylglucosamine & $1.0 \times 10^{4}$ & $1.3 \times 10^{4}$ \\
$\alpha_{1}$-Acid glycoprotein & 4.0 & $1.4-4.5$ \\
Fetuin & 60 & 21 \\
Bovin submaxillary mucin & $0.32-0.65$ & 0.32 \\
\hline
\end{tabular}

${ }^{a}$ Ref. (24). 
nose and galactose conjugates with Con $\mathrm{A}$ and Jacalin, respectively (11). Given the objective of the present study to use an array of lectin/enzyme-saccharide reagents, we initially pursued the synthesis of additional enzyme-saccharide structures that would be useful as indicators for the binding of other lectins. It is known that WGA lectin recognizes $N$-acetylglucosamine with high affinity (19), and thus a sequence of $\mathrm{MDH}$-glcNAc conjugates with varying amounts of glcNAc substitution was prepared. Among the resulting conjugates, the activity of an $\mathrm{MDH}$-glcNAc conjugate (prepared with an initial molar ration of saccharide to enzyme of 500 and determined to contain $12.3 \mathrm{glcNAc}$ residues per $\mathrm{MDH}$ after conjugation) was found to be inhibited up to $62 \%$ in the presence of excess WGA lectin. The kinetics of the binding reaction between WGA and this conjugate were examined by observing the percentage inhibition of conjugate after varying equilibration times with the added lectin (in excess, $100 \mu \mathrm{g} / \mathrm{ml}$ ). Maximum inhibition is observed after $20 \mathrm{~min}$, although the binding reaction is nearly complete after only $5 \mathrm{~min}$ of incubation (i.e., $>55 \%$ inhibition is still observed after this time period). Figure 1 shows the effect of varying amounts of WGA, as well as Con A and Jacalin, on the activity of a given amount of this $\mathrm{MDH}$-glcNAc conjugate using a 10-min incubation period between added lectin and conjugate. Note that over the concentration range examined, only WGA inhibits the activity of the conjugate. The degree of inhibition and binding kinetics of the new WGA/MDH-glcNAc homogeneous reagent system are quite comparable to that observed previously with the Con A/MDH-mannose and Jacalin/MDH-galactose reagents (11), and thus all three reagents systems are potentially useful to probe the carbohydrate structure/ content of intact glycoproteins.

Prior to examining native glycoproteins with the three lectin/MDH-saccharide reagent sets, model BSA-saccharide conjugates containing varying degrees of terminal mannose or galactose groups were used as test glycoproteins to determine whether the proposed approach can detect, semiquantitatively, the relative amount of carbohydrate within these synthetic glycoconjugates. Table 1 summarizes the results of this initial study. As shown, the $\mathrm{ED}_{50}$ values for each of the lectin/conjugate systems examined decreases in an amount proportional to the degree of saccharide substitution on the BSA.

To further test whether the lectin/enzyme-saccharide reagent sets could distinguish between variations of at least two different saccharides on the same protein, additional BSA conjugates with varying mannose/galactose molar ratios (approx $3 / 1,1 / 1$, and $1 / 3$ ) were prepared by reacting BSA with the same ratios of the respective $p$-isothiocyanatophenyl- $\alpha$-D-gycosides. The degree of total monosaccharide substitution for each conjugate was found to be between 26 and 30 , as deter- mined by the phenol-sulfuric acid method (17), with the ratio of BSA-appended carbohydrates (after extensive dialysis) presumed to be approximately equal to the starting conjugation ratio (note: galactose and mannose residues yield equivalent color in the phenol-sulfuric acid assay method). As shown in Table 2. there is a direct correlation between the competitive $\mathrm{ED}_{50}$ values observed with the Jacalin/MDH-galactose and Con A/ $\mathrm{MDH}$-mannose reagent systems, and the mannose/galactose ratio in these synthetic glycoconjugates. BSAsaccharide conjugates with higher mannose/galactose residues produce lower $\mathrm{ED}_{50}$ values with the Con $\mathrm{A} /$ $\mathrm{MDH}-$ mannose system and greater $\mathrm{ED}_{50}$ values with the Jacalin/MDH-galactose system. This is expected given the known binding specificity of the lectins.

We further investigated the use of proposed lectinbased homogeneous ELBA to detect the type and relative amount of carbohydrate structures within several native glycoproteins. By utilizing three different lectin/ MDH-saccharide assay systems, various dose-responses can be obtained for a given glycoprotein. For this purpose, four different glycoproteins (from commercial sources) containing saccharides with different ratios were employed as test proteins. The content of various carbohydrate groups in these proteins is summarized in Table 3 (literature values). Three different sets of dose-response curves were constructed by plotting percentage inhibition vs concentration of glycoprotein analyte. The results with the Con A/MDH-mannose and the Jacalin/MDH galactose systems are illustrated in Figs. 2 and 3. As shown, each protein exhibits a different dose-response behavior with each assay (i.e., effective detection range and steepness). In general, for the Con A system, the curves are shifted to lower detection ranges as the mannose content (wt\%) of the glycoproteins increase. Among the glycoproteins examined, $\beta$-fructofuranosidase, which has the highest mannose residue (see Table 3 ), yields the most sensitive dose-response curve with the Con $\mathrm{A} / \mathrm{MDH}$-mannose reagents. The curves for $\alpha$-amylase and $\alpha_{1}$-acid glycoprotein appear to be reversed from that expected based on total mannose content (see Table 3), although there is not a large difference in the relatively low weight percent of mannose for these two proteins. Clearly, the accessibility of lectin binding within the three-dimensional structure of the proteins is also an important factor in determining the relative response of the system to each test glycoprotein. In addition, the actual polysaccharide structure/sequence within the intact protein will also play an important role [some lectins may recognize terminal saccharides better than those within a branched or straight chain sequence (21)]. Another important factor influencing the dose-response behavior will be the ability of a given lectin, with multiple binding sites, to simultaneously bind two carbohydrate sites within the same glycoprotein. This effectively would increase 
the affinity of the lectin toward that glycoprotein (22) and dramatically increase the sensitivity of dose-response relative toward one where only single site binding was possible. The dose-response behavior of the four proteins with the Jacalin/MDH-galactose system is as expected, with $\alpha_{1}$-acid glycoprotein yielding the greatest response due to the high galactose content of this species (see Fig. 3).

Table 4 summarizes the $\mathrm{ED}_{50}$ values obtained for the different test proteins using the three lectin/MDH-saccharide reagent sets. On the basis of the $\mathrm{ED}_{50}$ values it is easy to distinguish those proteins that have an abundance of one type of saccharide structure and those that lack significant amounts of another. The WGA/MDHglcNAc system is also potentially useful for detecting glycoproteins with terminal sialic acid groups. Indeed, WGA is known to have binding affinity toward sialic acid, although somewhat less than its affinity for $N$ acetylglucosamine. This may be due to the structural similarity at positions $\mathrm{C}-2$ ( $\mathrm{N}$-acetamido group) and $\mathrm{C}-3$ (hydroxyl group) of the pyranose ring of both saccharide structures. These positions are known to be critical for WGA binding activity (23). Utilizing the binding property of WGA toward sialic acid, the WGA/MDHglcNAc system was employed to detect several sialic acid-rich glycoproteins (i.e., bovine submaxillary mucin and fetuin). Dose-response curves for these two glycoproteins show reasonable detection ranges (see Fig. 4). In addition, when these two proteins are used along with $N$-acetylglucosamine and $\alpha_{1}$-acid glycoprotein to test the performance of the proposed homogeneous assay system vs a standard WGA-based agglutinin method (24), the $\mathrm{ED}_{50}$ values of both systems are comparable (see Table 5). It should be noted that the classical agglutinin test employs red blood cells, requires $20 \mathrm{~min}$, and is, at best, only semiquantitative.

In summary, we have assessed the applicability of several lectin-based homogeneous enzyme-linked competitive binding assays for detecting the type and relative amount of specific carbohydrate structures within intact glycoproteins. For the most part, there is a direct correlation between known carbohydrate content of the glycoproteins and the responses to each within the different lectin/enzyme-saccharide conjugate systems. With appropriately prepared enzyme-saccharide conjugates, the method is simple and rapid and may offer an attractive alternative to existing techniques for the detection of the specific type and relative amounts of carbohydrate structures within glycoproteins. Future efforts will focus on devising additional homogeneous reagent sets, particularly suitable enzyme-sialic acid conjugates for more selective detection glycoproteins containing terminal sialic acid structures. In addition, investigations regarding the possibility of adapting these homogeneous assays to a 96 -well microtiter plate arrangement for more convenient and simultaneous evaluation of a large number of glycoproteins are in progress.

\section{ACKNOWLEDGMENTS}

1.B. gratefully acknowledges support in the form of a summer faculty fellowship from the Office of Research at the University of Michigan at Flint. This work was supported by grants from the National Science Foundation (CHE 8813952 and CHE 9119728).

\section{REFERENCES}

1. Spellman, M. W., Basa, L. J., Leonardo, C. K., Chakel, J. A., O'Conner, J. V., Wilson, S., and van Halbook, H. (1989) J. Biol. Chem. 264, 14,100-14,111.

2. Spellman, M. W. (1990) Anal. Chem. 62, 1714-1722.

3. Barr, J. R., Anumula, K. R., Vottese, M. B., Taylor, P. B., and Carr, S. A. (1991) Anal. Biochem. 192, 181-192.

4. Atkinson, P. H., Grey, A., Carver, J., Hakimi, J., and Ceccarini, C. (1981) Biochemistry 20, 3979-3986.

5. Ogata, S., Muramatsu, T., and Kobata, A. (1975) J. Biochem. 78, 687-696.

6. Green, E. D., and Baenziger, J. U. (1989) TIBS 14, 168-172.

7. Merkle, R. K., and Cummings, R. D. (1987) in Methods in Enzymology (Ginsberg, V., Ed.), Vol. 138, pp. 232-259, Academic Press, San Diego, CA.

8. Kinoshita, N., Suzuki, S., and Taniguchi, N. (1989) Clin. Chim. Acta 179, 143-152.

9. McCoy, J. P., Varani, J., and Goldstein, I. J. (1983) Anal. Biochem. 130, 437-444.

10. Egber, T. B., and Gottfried, A. F. (1989) Clin. Chem. 35, 2190 2195.

11. Kim, B. H., Cha, G. S., and Meyerhoff, M. E. (1990) Anal. Chem. 62, 2663-2668.

12. Ullman, E. F., and Maggio, E. T. (1980) in Enzyme-Immunoassay (Margio, E. T., Ed.), Chap. 5, CRC Press, Boca Raton, FL.

13. Henderson, D. R., Friedman, S. B., Harris, J. D., Manning, W. B., and Zoccoli, M. (1986) Clin. Chem. 32, 1637-1641.

14. Gibbons, I., Scold, C., Rowly, G. L., and Ullman, E. F. (1980) Anal. Biochem. 102, 167-170.

15. Amonta, R., Tarnowski, T., Gibbons, I., and Ullman, E. F. (1985) Anal. Biochem. 146, 211-219.

16. Schray, K. J., and Niedbala, R. S. (1988) Anal. Chem. 60, 353356.

17. Dubois, M., Gilles, K. A., Hamilton, J. K., Rebers, P. A., and Smith, F. (1956) Anal. Chem. 28, 350-356.

18. Reissig, J. L., Strominger, F. L., and Leloir, L. F. (1955) J. Biol. Chem. 217, 959-966.

19. Nagala, Y., and Burger, M. M. (1974) J. Biol. Chem. 249, 31163122.

20. Gottschalk, A. (1972) Glycoproteins: Their Composition, Structure, and Function, Elsevier, Amsterdam.

21. Skea, D. D., Christopoulou, S. P., Plaut, A. G., and Underdown, B. J. (1988) Mol. Immunol. 25, 1-6.

22. Aucouturia, P., Mihaesco, E., Minaesco, C., and Preud'Homme, J. P. (1987) Mol. Immunol. 24, 503-511.

23. Goldstein, I. J. (Ed.) (1986) The Lectins: Properties, Functions, and Applications in Biology and Medicine, Academic Press, New, York.

24. Bhavanandan, V. P., and Katlic, A. W. (1979) J. Biol. Chem. 254, 4000-4008. 